This item was submitted to Loughborough's Research Repository by the author.

Items in Figshare are protected by copyright, with all rights reserved, unless otherwise indicated.

\title{
Detailed wave function analysis for multireference methods: Implementation in the molcas program package and applications to tetracene
}

\section{PLEASE CITE THE PUBLISHED VERSION}

https://doi.org/10.1021/acs.jctc.7b00718

\section{PUBLISHER}

(C) American Chemical Society (ACS)

\section{VERSION}

AM (Accepted Manuscript)

\section{PUBLISHER STATEMENT}

This work is made available according to the conditions of the Creative Commons Attribution-NonCommercialNoDerivatives 4.0 International (CC BY-NC-ND 4.0) licence. Full details of this licence are available at: https://creativecommons.org/licenses/by-nc-nd/4.0/

\section{LICENCE}

CC BY-NC-ND 4.0

\section{REPOSITORY RECORD}

Plasser, Felix, Stefanie A. Mewes, Andreas Dreuw, and Leticia Gonzalez. 2019. "Detailed Wave Function Analysis for Multireference Methods: Implementation in the Molcas Program Package and Applications to Tetracene". figshare. https://hdl.handle.net/2134/32227. 


\title{
Detailed wavefunction analysis for multireference methods: Implementation in the Molcas program package and applications to tetracene
}

\author{
Felix Plasser, ${ }^{*}, \dagger$ Stefanie A. Mewes, ${ }^{\ddagger} \uparrow$ Andreas Dreuw, ${ }^{\ddagger}$ and Leticia González ${ }^{\dagger}$ \\ $\dagger$ Institute for Theoretical Chemistry, Faculty of Chemistry, University of Vienna, \\ Währingerstr. 17, 1090 Vienna, Austria \\ $\ddagger$ Interdisciplinary Center for Scientific Computing, Heidelberg University, Im Neuenheimer \\ Feld 205 A, 69120 Heidelberg, Germany \\ ICentre for Theoretical Chemistry and Physics, The New Zealand Institute for Advanced \\ Study (NZIAS), Massey University Albany, Private Bag 102904, Auckland 0745, New \\ Zealand \\ E-mail: felix.plasser@univie.ac.at
}

This document is the Accepted Manuscript version of a Published Work that appeared in final form in Journal of Chemical Theory and Computation, copyright (c) American Chemical Society after peer review and technical editing by the publisher. To access the final edited and published work see: http://pubs.acs.org/articlesonrequest/AORQkGJHHR7rW7YztpZgJXC

\section{Abstract}

High-level multireference computations on electronically excited and charged states of tetracene are performed, and the results are analyzed using an extensive wavefunction analysis toolbox that has been newly implemented in the MOLCAS program package. Aside from verifying the strong effect of dynamic correlation, this study reveals an unexpected critical influence of the atomic orbital basis set. It is shown that different polarized double- $\zeta$ basis sets produce significantly different results for energies, densities and overall wavefunctions, with the best performance obtained for the atomic natural orbital (ANO) basis set by Pierloot et al. Strikingly, the ANO basis set does not only reproduce the energies but also performs exceptionally well in terms of describing the diffuseness of the different states and of their attachment/detachment densities. This study, thus, underlines the fact that diffuse basis functions are needed for an accurate description of the electronic wavefunctions but also shows that, at least for the present example, it is enough to include them implicitly in the contraction scheme.

\section{Introduction}

Recent years have seen a surge in the development of multireference methods and it is possible nowadays to compute the electronic structure of large systems containing many active electrons, interacting orbitals, and quasidegenerate states. ${ }^{1-7}$ However, the results of such calculations crucially depend on the appropriate choice of the wavefunction parameters, such as the one-electron basis set, the active orbital space, and the inclusion of dynamic 
correlation. Also more specific parameters, that are not even in principle amenable to systematic improvement, such as the empirical IPEA shift in complete active space perturbation theory $(\mathrm{CASPT} 2)^{8}$ or approximate extensivity corrections in multireference configuration interaction can strongly affect the calculations. ${ }^{9-11}$ Tuning the parameters usually occurs in a brute-force approach where the parameters are varied until the energies coincide with a computational or experimental reference. More refined protocols, see e.g. Ref. 12, are only applied rarely.

For successfully performing multireference computations it is essential to obtain a detailed understanding of the wavefunctions produced. However, this can be a formidable challenge, requiring patience as well as expert knowledge, since it is often necessary to disentangle the effects of many interacting electronic configurations and orbitals. And even if a qualitative understanding of the wavefunctions can be obtained, it is problematic that much of the discussion relies on subjective reasoning, e.g. manual assignment of orbital character. Consequently, if something goes wrong, it is difficult to identify the underlying methodological issues. To overcome this problem, it is highly desirable to have well-defined quantitative analysis methods able to provide deeper insight into the wavefunctions produced in modern multireference calculations.

Recently, some of us have developed the software library LIBWFA, ${ }^{13}$ which provides quantitative and visual wavefunction analysis tools allowing to study diverse phenomena, such as localization and charge transfer of electronic excitations, ${ }^{14}$ double excitation character, ${ }^{15}$ and secondary orbital relaxation. ${ }^{16}$ In addition, an exciton analysis formalism has been introduced $^{17}$ that provides detailed real space insight into excitation processes and allows to analyze excited states in terms of correlation ${ }^{18}$ and entanglement. ${ }^{19}$ These methods have been employed successfully to study diverse processes such as exciton formation in conjugated polymers, ${ }^{20,21}$ charge transfer in donor-acceptor compounds, ${ }^{22,23}$ electronic excitations in transition metal complexes, ${ }^{24}$ core excitations, ${ }^{25}$ and two-photon absorption. ${ }^{26}$ However, in all this previous work only single-reference methods were employed. Within this work, the wavefunction analysis procedures are extended to a multireference framework. To this aim we constructed an interface between the LIBWFA library and the program system MoLCAS. Using this new interface it is possible to analyze multireference wavefunctions constructed at the complete and restricted active space self-consistent field (CASSCF/RASSCF) levels of theory, including state mixing effects derived from multistate perturbation theory (MSCASPT2).

The new analysis methods are used here to provide detailed insight into various charged and excited states of tetracene and to see how their description is affected by the computational methodology. Tetracene is chosen as a representative of the polyacenes, a substance class playing a central role in modern organic electronics as versatile organic semiconductors $^{27}$ and field-effect transistors. ${ }^{28}$ The application of polyacenes in photovoltaics ${ }^{29,30}$ and especially their suitability for singlet fission $^{31-33}$ has attracted significant attention. Aside from a wide range of experimental studies, ${ }^{34-36}$ computation plays a crucial role in elucidating the electronic structure properties that form the basis for the exceptional performance of polyacenes in organic electronics. The urge to explain these properties has inspired numerous contemporary investigations of polyacene excited states, their charge transport characteristics as well as their groundstate properties. The singlet and triplet excited states of polyacenes ${ }^{37-40}$ including double excitations ${ }^{41-43}$ and charge transfer (CT) states ${ }^{44,45}$ as well as the ionization potentials and electron affinities, ${ }^{46,47}$ charge transport properties, ${ }^{48-51}$ and excited states of the radical cations ${ }^{41}$ have been investigated. The neutral ground states possess unexpected properties and have been scrutinized with the aim of determining unpaired electrons and multireference character. ${ }^{4,42,43,52-56}$ Specifically, in the case of singlet fission a range of electronic structure computations $^{57-61}$ and dynamics simulations ${ }^{62,63}$ were carried out. Singlet fission is a particularly challenging case for electronic structure theory as 
it requires an accurate description of the locally excited singlet and triplet, as well as the charge transfer states to provide a correct description of the relevant channels, i.e. the direct and charge transfer mediated ${ }^{31}$ mechanisms.

A particularly challenging aspect in the description of the excited states of polyacenes is that the results critically depend on the electronic structure method used. Previously, an accurate treatment of dynamic electron correlation has been shown to be required for wavefunction based methods ${ }^{60,64}$ and an inclusion of non-local exchange for time-dependent density functional theory (TDDFT). ${ }^{37,39,65}$ In the present study, it is shown that also the oneelectron basis set plays a crucial role in the description of the lowest singlet and triplet excitation energies, as well as the ionization potential (IP) and electron affinity (EA) of tetracene.

\section{Wavefunction Analysis Methodology}

We employ different strategies to characterize electronic wavefunctions in order to understand what changes between different electronic states and how the results of computations are affected by the methodology. First, we investigate the overall spatial extent of the electron distribution in order to quantify how the electron density contracts or expands with the removal or addition of an electron. For this purpose, the root-mean-square (RMS) size of the electron density $\rho$ is computed as

$$
\sigma_{\rho}^{2}=N^{-1} \int \rho(r)\left(\vec{x}-\vec{m}_{\rho}\right)^{2} d r,
$$

where $N$ is the number of electrons and $\vec{x}$ is a vector containing the Cartesian coordinates. The vector $\vec{m}_{\rho}$ pointing to the center of electronic charge is defined as

$$
\vec{m}_{\rho}=N^{-1} \int \rho(r) \vec{x} d r .
$$

Eq. (1) is evaluated in terms of its individual components

$$
\sigma_{\rho}^{2}={\sigma_{\rho, x}}^{2}+{\sigma_{\rho, y}{ }^{2}+\sigma_{\rho, z}^{2}}^{2}
$$

These terms are in turn computed as

$$
\sigma_{\rho, x}{ }^{2}=N^{-1} \operatorname{tr}\left(\mathbf{D} \mathbf{M}_{x x}\right)-\left[N^{-1} \operatorname{tr}\left(\mathbf{D} \mathbf{M}_{x}\right)\right]^{2}
$$

where $\mathbf{D}$ is the density matrix of the state of interest, and $\mathbf{M}_{x}$ and $\mathbf{M}_{x x}$ are the dipole and quadrupole moment integrals. To put this quantity in perspective, it is worth noting that for a hydrogen atom the $\sigma_{\rho, x}, \sigma_{\rho, y}$, and $\sigma_{\rho, z}$ values are per definition 1 Bohr radius.

A possible downside of the above analysis is that it is dominated by the bulk of the electrons, which do not undergo changes during an electronic transition. Therefore, as a second option, we analyze the one-particle difference density matrix (1DDM) of the state of interest with respect to the neutral ground state, which is a more sensitive measure for changes in the electronic structure. The 1DDM is constructed by subtracting the density matrix of the ground state $\mathbf{D}^{00}$ from the one of the excited state $\mathbf{D}^{I I}$. Subsequently the attachment/detachment analysis of Head-Gordon et al. ${ }^{66}$ can be performed. This procedure starts with a diagonalization of the difference density matrix

$$
\mathbf{W}^{\mathrm{T}}\left(\mathbf{D}^{I I}-\mathbf{D}^{00}\right) \mathbf{W}=\operatorname{diag}\left(\kappa_{1}, \kappa_{2}, \ldots\right)
$$

where the $\kappa_{i}$ are the eigenvalues of the 1DDM, and $\mathbf{W}$ is the matrix containing the eigenvectors, termed natural difference obitals (NDOs). ${ }^{15}$ The NDOs can be used to visualize the excitation process ${ }^{67}$ similarly to the natural transition orbitals (NTO) while also accounting for many-body and orbital-relaxation effects. ${ }^{16,24}$ The detachment density matrix $\mathbf{D}_{D}$ is obtained by considering the negative eigenvalues of the $1 \mathrm{DDM}$

$$
d_{i}=\min \left(\kappa_{i}, 0\right)
$$

and transforming back to the initial orbital basis

$$
\mathbf{D}_{D}=\mathbf{W} \operatorname{diag}\left(d_{1}, d_{2}, \ldots\right) \mathbf{W}^{\mathrm{T}} .
$$

Likewise, the attachment density $\mathbf{D}_{A}$ is con- 
structed from the positive eigenvalues using

$$
\begin{aligned}
a_{i} & =\max \left(\kappa_{i}, 0\right) \\
\mathbf{D}_{A} & =\mathbf{W} \operatorname{diag}\left(a_{1}, a_{2}, \ldots\right) \mathbf{W}^{\mathrm{T}} .
\end{aligned}
$$

After these transformations, it is possible to visualize the attachment and detachment densities in coordinate space, which is done below.

The traces of the attachment and detachment matrices

$$
\begin{aligned}
& p_{D}=-\operatorname{tr}\left(\mathbf{D}_{D}\right)=-\sum_{i} d_{i} \\
& p_{A}=\operatorname{tr}\left(\mathbf{D}_{A}\right)=\sum_{i} a_{i},
\end{aligned}
$$

called promotion numbers, ${ }^{66}$ count the total number of detached and attached electrons. For a neutral excitation $p_{D}$ is equal to $p_{A}$ while in the case of an ionization process, the difference corresponds to the number of electrons gained or lost. In addition, it is possible to quantify the number of orbitals involved in the attachment and detachment processes using the detachment and attachment participation ratios (PR) defined as ${ }^{15}$

$$
\mathrm{PR}_{D}=\frac{p_{D}^{2}}{\sum_{i} d_{i}^{2}} \quad \mathrm{PR}_{A}=\frac{p_{A}^{2}}{\sum_{i} a_{i}^{2}}
$$

The $\mathrm{PR}_{D}$ value is equal to 1 , if there is only one non-vanishing eigenvalue $\left(d_{1}=p_{D}\right)$. In the case of $k$ non-vanishing eigenvalues of equal magnitude the $\mathrm{PR}_{D}$ value is equal to $k$. For more general cases $\mathrm{PR}_{D}$ and the analogous $\mathrm{PR}_{A}$ can be understood as an effective number of involved orbitals in the detachment and attachment processes. For a related discussion see Ref. 19.

As a final descriptor, we quantify the spatial extent of the attachment and detachment densities in analogy to Eq. (1), using, e.g., for the $x$-component of the attachment density

$$
\sigma_{A, x}{ }^{2}=p_{A}^{-1} \operatorname{tr}\left(\mathbf{D}_{A} \mathbf{M}_{x x}\right)-\left[p_{A}^{-1} \operatorname{tr}\left(\mathbf{D}_{A} \mathbf{M}_{x}\right)\right]^{2}
$$

and analogous equations for the other Cartesian components and the detachment density.

One should note that the above methods rely on operator integrals and analyses of eigenvalue spectra. Since no population analysis methods are used here, the approach is particularly wellsuited to evaluate the effects of different basis sets in an unbiased manner.

\section{Computational Details}

Computations were performed using $D_{2 h}$ symmetry with the tetracene molecule located in the $x y$-plane and the long molecular axis arranged along the $y$-axis. The geometry of tetracene was optimized in the ground state at the MP2/def2-TZVP level of theory and kept fixed for all subsequent computations (see Table $\mathrm{S} 1$ for the coordinates). Complete active space self-consistent field (CASSCF) ${ }^{68}$ singlepoint computations were performed using $12 \mathrm{ac}$ tive $\pi$-orbitals $\left(3 \times b_{2 g}, 3 \times b_{3 g}, 3 \times a_{u}, 3 \times b_{1 u}\right)$ occupied by 11,12 , and 13 electrons for the cationic, neutral, and anionic states, respectively. All computed states are characterized uniquely by either spatial symmetry, spin or particle number. Therefore, no state averaging was performed but all states were computed with respect to their individually optimized orbitals. Dynamic correlation effects were incorporated by second-order perturbation theory (CASPT2).${ }^{8}$ For the IPEA shift,${ }^{69}$ two different values, 0 and 0.25 a.u., were investigated. The Cholesky decomposition of the two-electron repulsion integrals was employed. ${ }^{70}$

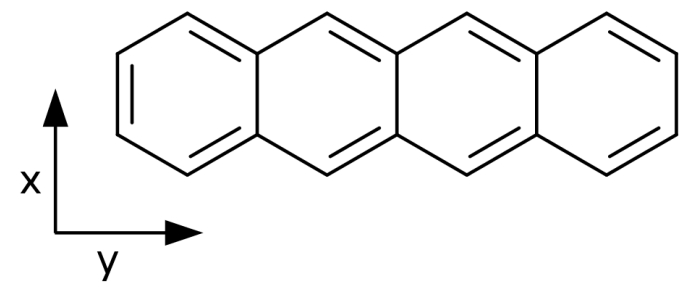

Scheme 1: Molecular structure of the tetracene molecule and definition of the coordinate axes.

Various atomic orbital (AO) basis sets, shown in Table 1, were employed in connection with these methods: the unpolarized and polarized Pople double- $\zeta$ basis sets 6-31G and 6$31 \mathrm{G}^{* *},{ }^{71,72}$ the latter basis set with modified exponents for the polarization functions 
$6-31 G^{* *}(0.25,0.15)$ as introduced by KroonBatenburg ${ }^{73}$ and popularized by Hobza and coworkers, ${ }^{74}$ three additional double- $\zeta$ basis sets SVP, ${ }^{75}$ cc-pVDZ, ${ }^{76}$ and ANO-S-VDZP, ${ }^{77}$ and the increasingly larger basis sets aug-ccpVDZ, ${ }^{78}$ ANO-L-VTZP, ${ }^{79}$ aug-cc-pVTZ. ${ }^{78}$ For the following discussion it is important to notice that the five polarized double- $\zeta$ basis sets have the same number of contracted basis functions (see Table 1) and that, consequently, their computational cost is identical aside from the initial computation of the AO integrals. At the same time, these basis sets differ significantly in the number and coefficients of the primitive basis functions and, thus, in the wavefunctions produced. It is worth noting that ANO-S-VDZP includes primitive $s$ and $p$ basis functions with exponents of $\zeta=0.055$ and 0.043 a.u. that are about as diffuse as the diffuse $s$ and $p$ functions of aug-cc-pVDZ, and that the most diffuse $d$ functions of ANO-S-VDZP $(\zeta=0.092$ a.u.) are even more diffuse than in the aug-cc-pVDZ case. On the other hand, these diffuse exponents are only included implicitly in the ANOS-VDZP contraction scheme and their role is not immediately clear. The question whether ANO-S-VDZP does indeed behave similarly to a diffuse basis set will be investigated below by considering energies, wavefunctions, and densities.

The wavefunctions were analyzed using the methods described in Section 2 by employing the newly created interface between the wavefunction analysis library LIBWFA ${ }^{13}$ and the MOLCAS program package. ${ }^{80}$ This interface works through exchanging all relevant data in the form of an HDF5 (hierarchical data format) file, thus allowing for a flexible and modular approach of the analysis and postprocessing steps. Technically, the 1DMs were computed through the CAS state interaction method $^{81}$ as implemented in the RASSI program of the MolcAs suite. The calculations were performed using a development version of the Molcas 8.1 package, and the functionality described will be made available within the open-source OPENMOLCAS package in the near future. Post-processing was achieved through the Molpy ${ }^{82}$ and TheoDORE ${ }^{83}$ codes. Plot- ting of densities was performed by means of the dgrid code ${ }^{84}$ in connection with the VMD program package. ${ }^{85}$

Aside from density matrix analyses, we use wavefunction overlaps to compare the CASSCF wavefunctions constructed over different basis sets, following Ref. 86. This formalism starts by computing the overlaps between the AOs of the different basis sets and continues by computing the overlap between molecular orbitals (MO) of the two computations. Subsequently for every pair of bra and ket Slater determinants, it is necessary to compute the determinant of the matrix containing all mutual $\mathrm{MO}$ overlaps. This last step can be exceedingly expensive requiring, e.g., the computation of $4.6 \times 10^{10}$ such terms for $\operatorname{CASSCF}(12,12)$ singlet states. To overcome this computational bottleneck, the algorithm proposed in Ref. 87 was employed, which allows for the extensive use of recurring intermediates and, thus, makes such calculations feasible. The corresponding code is distributed as a part of the SHARC molecular dynamics suite. ${ }^{88-90}$

\section{Results}

In this work, we investigate the effects of different wavefunction parameters on the energies and wavefunctions of charged and neutral states of tetracene. Specifically, we consider all possible states obtained through one-electron processes involving the formal highest occupied molecular orbital (HOMO) and the lowest unoccupied MO (LUMO). This gives rise to the singlet and triplet coupled neutral excitations: the $S_{1}\left(L_{a}\right)$ and the $T_{1}$ state, both of $B_{3 u}$ symmetry, as well as the ground state of the cation of $A_{u}$ symmetry $\left(D_{0}^{+}\right)$and the ground state of the anion of $B_{3 g}$ symmetry $\left(D_{0}^{-}\right)$. The choice was made for these four states due to their close relations in a one-electron picture as well as the fact that these states are invoked in the different mechanisms used to explain singlet fission.

In the following we consider the excitation energies of the singlet and triplet states, denoted $\Delta E\left(S_{1}\right)$ and $\Delta E\left(T_{1}\right)$, as well as the ionization potential (IP) and electron affinity (EA). In ad- 
Table 1: Basis sets used in this study: contraction scheme for the $\mathrm{C}$ and $\mathrm{H}$ atoms, total number of primitive/contracted basis functions, and primary literature references.

\begin{tabular}{lcccl}
\hline \hline Basis set & $\mathrm{C}$ & $\mathrm{H}$ & Total & Refs \\
\hline 6-31G & $(10 \mathrm{~s} 4 \mathrm{p}) /[3 \mathrm{~s} 2 \mathrm{p}]$ & $(4 \mathrm{~s}) /[2 \mathrm{~s}]$ & $444 / 186$ & 71 \\
6-31G** & $(10 \mathrm{~s} 4 \mathrm{p} 1 \mathrm{~d}) /[3 \mathrm{~s} 2 \mathrm{p} 1 \mathrm{~d}]$ & $(4 \mathrm{~s} 1 \mathrm{p}) /[2 \mathrm{~s} 1 \mathrm{p}]$ & $570 / 312$ & 71,72 \\
6-31G** $(0.25,0.15)$ & $(10 \mathrm{~s} 4 \mathrm{p} 1 \mathrm{~d}) /[3 \mathrm{~s} 2 \mathrm{p} 1 \mathrm{~d}]$ & $(4 \mathrm{~s} 1 \mathrm{p}) /[2 \mathrm{~s} 1 \mathrm{p}]$ & $570 / 312$ & 73 \\
SVP & $(7 \mathrm{~s} 4 \mathrm{p} 1 \mathrm{~d}) /[3 \mathrm{~s} 2 \mathrm{p} 1 \mathrm{~d}]$ & $(4 \mathrm{~s} 1 \mathrm{p}) /[2 \mathrm{~s} 1 \mathrm{p}]$ & $516 / 312$ & 75 \\
cc-pVDZ & $(9 \mathrm{~s} 4 \mathrm{p} 1 \mathrm{~d}) /[3 \mathrm{~s} 2 \mathrm{p} 1 \mathrm{~d}]$ & $(4 \mathrm{~s} 1 \mathrm{p}) /[2 \mathrm{~s} 1 \mathrm{p}]$ & $552 / 312$ & 76 \\
ANO-S-VDZP & $(10 \mathrm{~s} 6 \mathrm{p} 3 \mathrm{~d}) /[3 \mathrm{~s} 2 \mathrm{p} 1 \mathrm{~d}]$ & $(7 \mathrm{~s} 3 \mathrm{p}) /[2 \mathrm{~s} 1 \mathrm{p}]$ & $966 / 312$ & 77 \\
aug-cc-pVDZ & $(10 \mathrm{~s} 5 \mathrm{p} 2 \mathrm{~d}) /[4 \mathrm{~s} 3 \mathrm{p} 2 \mathrm{~d}]$ & $(5 \mathrm{~s} 2 \mathrm{p}) /[3 \mathrm{~s} 2 \mathrm{p}]$ & $762 / 552$ & 76,78 \\
ANO-L-VTZP & $(14 \mathrm{~s} 9 \mathrm{p} 4 \mathrm{~d} 3 \mathrm{f}) /[4 \mathrm{~s} 3 \mathrm{p} 2 \mathrm{~d} 1 \mathrm{f}]$ & $(8 \mathrm{~s} 4 \mathrm{p} 3 \mathrm{~d}) /[3 \mathrm{~s} 2 \mathrm{p} 1 \mathrm{~d}]$ & $1896 / 708$ & 79 \\
aug-cc-pVTZ & $(11 \mathrm{~s} 6 \mathrm{p} 3 \mathrm{~d} 2 \mathrm{f}) /[5 \mathrm{~s} 4 \mathrm{p} 3 \mathrm{~d} 2 \mathrm{f}]$ & $(6 \mathrm{~s} 3 \mathrm{p} 2 \mathrm{~d}) /[4 \mathrm{~s} 3 \mathrm{p} 2 \mathrm{~d}]$ & $1344 / 1104$ & 76,78 \\
\hline \hline
\end{tabular}

dition, two derived quantities will be discussed. Firstly, the fundamental gap is computed

$$
E_{\text {gap }}=\mathrm{IP}-\mathrm{EA}
$$

which represents the energy required to remove an electron from one tetracene molecule and place it at a second molecule at infinite distance. When $E_{\text {gap }}$ is available, the energy of a CT state can be estimated as

$$
E_{\mathrm{CT}}(R)=E_{\text {gap }}-1 / R
$$

where $R$ is the separation between the molecules, cf. Ref. 91. In this sense, knowing $E_{\text {gap }}$ allows one to predict whether a method will yield appropriate energies for CT states without having to perform a supermolecular excited state calculation. As a second quantity of interest, we compute the characteristic energy for singlet fission as

$$
E_{\mathrm{stt}}=2 \times \Delta E\left(T_{1}\right)-\Delta E\left(S_{1}\right) .
$$

A positive $E_{\text {stt }}$ corresponds to endothermic singlet fission (in a vertical approximation) while a negative value corresponds to an exothermic process.

Table 2 collects the energies discussed above computed for a number of methods. Specifically, we investigate the effect of three parameters in CASPT2 computations, (i) the active space, (ii) the one-electron basis set, and (iii) the IPEA shift. ${ }^{69}$ In addition, the general influence of dynamic correlation is dis- cussed by comparing the CASPT2 to the CASSCF results. The highest level used here, CASPT2(12,12)/aug-cc-pVTZ gives excellent agreement with the experiment if an IPEA shift of 0.25 a.u. is used. The $S_{1}$ energy, as well as the EA and IP are within $0.1 \mathrm{eV}$ of the experimental gas-phase reference. To the best of our knowledge, no experimental gas-phase reference for the $T_{1}$ state was reported so far. If one assumes that, similarly to the singlet, the gas-phase triplet energy is about $0.5 \mathrm{eV}$ above the energy in the crystal then the agreement is also good in this case.

Lowering the active space to the CASPT2 $(6,6) /$ augcc-pVTZ level has a notable effect only on the $T_{1}$ state, which is shifted up by $0.2 \mathrm{eV}$. This increase, however, leads to a significantly enhanced characteristic energy for singlet fission, $E_{\text {stt }}=0.88 \mathrm{eV}$. Next, the effects of lowering the basis set to the polarized double- $\zeta$ level are investigated considering the $6-31 \mathrm{G}^{* *}$ and ANOS-VDZP basis sets. Both basis sets contain the same number of contracted basis functions and thus require the same effort in the CASSCF and CASPT2 steps. However, drastic differences are found between those two basis sets. In the case of the ANO-S-VDZP basis set all values stay within $0.25 \mathrm{eV}$ of the aug-cc-pVTZ reference whereas significantly enhanced errors are observed for $6-31 \mathrm{G}^{* *}$. While the errors for $\Delta E\left(S_{1}\right)(0.35 \mathrm{eV})$ and the IP $(0.5 \mathrm{eV})$ still produce qualitatively reasonable results, the EA is underestimated by $0.9 \mathrm{eV}$ yielding only a barely positive value at all. The errors in the 
Table 2: Energies (given in eV) of neutral and charged states of tetracene computed using different methods: Excitation energies of the first singlet and triplet excited states $\left[\Delta E\left(S_{1}\right), \Delta E\left(T_{1}\right)\right]$, ionization potential (IP), electron affinity (EA), fundamental gap $\left(E_{\text {gap }}\right)$, and characteristic energy for singlet fission $\left(E_{\mathrm{stt}}\right)$.

\begin{tabular}{lll|llll|ll}
\hline \hline Method & IPEA & Basis set & $\Delta E\left(S_{1}\right)$ & $\Delta E\left(T_{1}\right)$ & IP & EA & $E_{\text {gap }}$ & $E_{\text {stt }}$ \\
\hline Experiment & & & $2.88^{a},\left(2.32^{b}\right)$ & $\left(1.25^{b}\right)$ & $6.97^{c}$ & $1.04^{d}$ & $5.93^{c, d}$ & $\left(0.18^{b}\right)$ \\
\hline CASPT2(12,12) & 0.25 & aug-cc-pVTZ & 2.76 & 1.62 & 6.92 & 0.99 & 5.93 & 0.49 \\
CASPT2(6,6) & 0.25 & aug-cc-pVTZ & 2.74 & 1.81 & 6.93 & 1.01 & 5.92 & 0.88 \\
CASPT2(12,12) & 0.25 & ANO-S-VDZP & 2.87 & 1.64 & 6.78 & 0.74 & 6.04 & 0.41 \\
CASPT2(12,12) & 0.25 & 6-31G** & 3.11 & 1.74 & 6.42 & 0.10 & 6.32 & 0.25 \\
CASPT2(12,12) & 0.00 & aug-cc-pVTZ & 2.37 & 1.22 & 6.77 & 1.13 & 5.64 & 0.08 \\
CASSCF $(12,12)$ & - & aug-cc-pVTZ & 4.12 & 1.76 & 6.18 & -0.73 & 6.90 & -0.60 \\
\hline \hline
\end{tabular}

${ }^{a}$ Refs 37,92: estimated vertical gas-phase excitation,

${ }^{b}$ Ref. 93: 0-0 transition (crystal),

${ }^{c}$ Ref. 94: vertical gas-phase IP, ${ }^{d}$ Ref. 95: gase-phase EA.

IP and EA partially cancel, but also $E_{\text {gap }}$ is overestimated by $0.4 \mathrm{eV}$ with the $6-31 \mathrm{G}^{* *}$ basis set, meaning that according to Eq. (15) the energy of CT states would be overestimated by this amount. Omitting the IPEA shift, which is included in the CASPT2 zeroth-order Hamiltonian by ad hoc reasoning, ${ }^{69}$ yields quite notably altered results. The $S_{1}$ and $T_{1}$ energies are shifted down by $0.4 \mathrm{eV}$ while also the IP and EA are affected. Incidentally, the unscaled CASPT2(12,12) data agree with the values reported from measurements in the solid state ${ }^{93}$ but this should only be seen as a coincidence. The most dramatic effect is observed in the CASSCF case, i.e. when omitting dynamic correlation. In this case the $S_{1}$ energy and EA are shifted by more than $1 \mathrm{eV}$, the IP is altered by $0.7 \mathrm{eV}$ and only the $T_{1}$ energy remains roughly constant. While the IP and EA are shifted in the same direction, a stronger effect on the EA leads to a rise of $E_{\text {gap }}$ by $1.0 \mathrm{eV}$ compared to CASPT2. Thus, CASSCF and related methods, which do not include dynamic correlation, can strongly overestimate the energies of $\mathrm{CT}$ states. A related effect has been discussed for the configuration interaction singles method. ${ }^{96}$

Summarizing the challenges in the computation of the different states of tetracene: An inclusion of dynamic correlation is necessary even for qualitatively meaningful results and the precise way to do so, i.e. the value of the IPEA shift, can still introduce errors of $0.5 \mathrm{eV}$. Notably, also the choice of the AO basis set plays an unexpectedly strong role. As the effect of dynamic correlation ${ }^{64}$ as well as the IPEA shift ${ }^{11}$ have been addressed recently in related systems, we will focus on the effects of the basis sets.

Excitation energies as well as the IP and EA were computed for the basis sets listed in Table 1 at the CASPT2(12,12) level. The results are plotted in Figure 1. In Figure 1 (a), the $S_{1}$ and $T_{1}$ energies are shown for different basis sets. For the chosen order of the basis sets a steep decline of the $S_{1}$ energies is observed while the $T_{1}$ energies are more or less constant. The values for the $6-31 \mathrm{G}$ basis set, which is the only one that does not include polarization functions, are unsurprisingly separated strongly from the remaining values. It is, however, remarkable that also between the different polarized double- $\zeta$ basis sets (i.e. going from $6-31 \mathrm{G}^{* *}$ to ANO-S-VDZP) a decrease of $0.28 \mathrm{eV}$ in excitation energies is observed. Interestingly, this difference is larger than the change of $0.13 \mathrm{eV}$ observed when going from ANO-SVDZP to aug-cc-pVTZ, which is probably close to the basis-set limit. The $6-31 \mathrm{G}^{* *}(0.25,0.15)$ basis set, which uses modified $d$-function exponents, provides significantly improved $S_{1}$ energies when compared to the original $6-31 \mathrm{G}^{* *}$ basis set. However, it is also worth pointing 


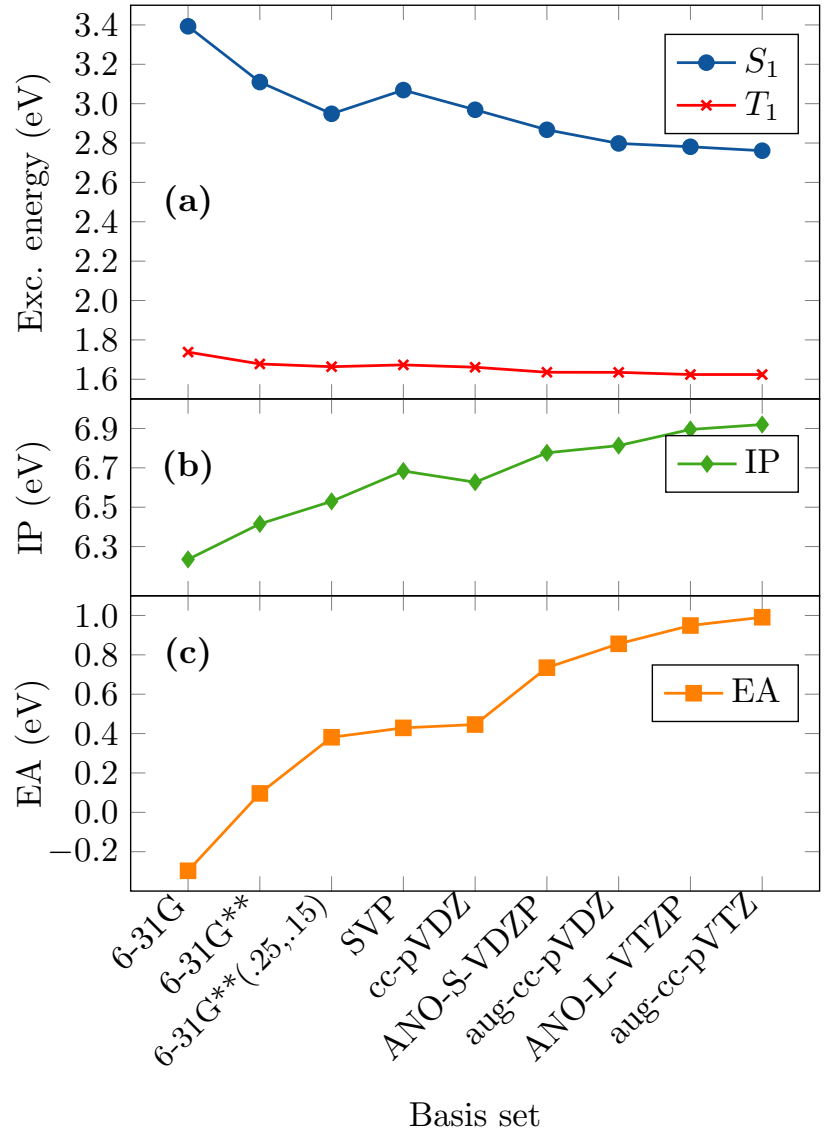

Figure 1: Vertical excitation energies (a), ionization potential (b), and electron affinity (c) of tetracene computed at the CASPT2 $(12,12)$ level $(\mathrm{IPEA}=0.25)$ using various basis sets. out that this improvement comes at the cost of shifting up the total CASPT2 energy by 0.5 a.u. (cf. Figure S5). Strong basis-set effects are also observed in the case of the IP (Figure $1(\mathrm{~b}))$. The unpolarized $6-31 \mathrm{G}$ basis is separated from the remaining results, but also the polarized $6-31 \mathrm{G}^{* *}$ and $6-31 \mathrm{G}^{* *}(0.25,0.15)$ basis sets are quite far away from the basisset limit, underestimating the IP by 0.5 and $0.4 \mathrm{eV}$, respectively. By comparison the SVP and cc-pVDZ results are significantly better, lying within about $0.3 \mathrm{eV}$ of the aug-cc-pVTZ result. ANO-S-VDZP and the larger basis sets move to within $0.15 \mathrm{eV}$ of this result. The EA [Figure 1 (c)] shows similar trends as compared to the IP although the errors are more severe. The 6-31G basis set fails to produce a positive EA at all while in the case of $6-31 \mathrm{G}^{* *}$ the electron is only loosely bound by $0.10 \mathrm{eV}$. The results are somewhat improved in the case of $6-31 \mathrm{G}^{* *}(0.25,0.15)$, SVP, and cc-pVDZ, yielding an EA of about $0.4 \mathrm{eV}$, which is, however, still less than half of the converged value. Figure 1 reveals a significant increase in performance when moving to the ANO-S-VDZP basis set (possessing the same number of contracted basis functions as the last four basis sets discussed), obtaining an EA of $0.86 \mathrm{eV}$, which is already close to the aug-cc-pVTZ result of $0.99 \mathrm{eV}$.

As a next step, we investigate the convergence of the wavefunctions with respect to the basis set following the strategies developed in Refs 86,87. For this purpose, the $\operatorname{CASSCF}(12,12)$ wavefunctions constructed with the different basis sets are projected against the aug-ccpVTZ reference and the corresponding weights (i.e. the squared overlap values) are plotted in Figure 2. Starting from the right, it is observed that the ANO-L-VTZP wavefunctions are almost identical to the aug-cc-pVTZ ones for all five states with squared overlaps around 0.995 indicating that these are indeed almost converged results. In the case of aug-cc-pVDZ and ANO-S-VDZP the squared overlap values are again very uniformly distributed around 0.97 and 0.96 , respectively. For the basis sets with a smaller number of primitive basis functions, the lines separate. Generally, the ground state 


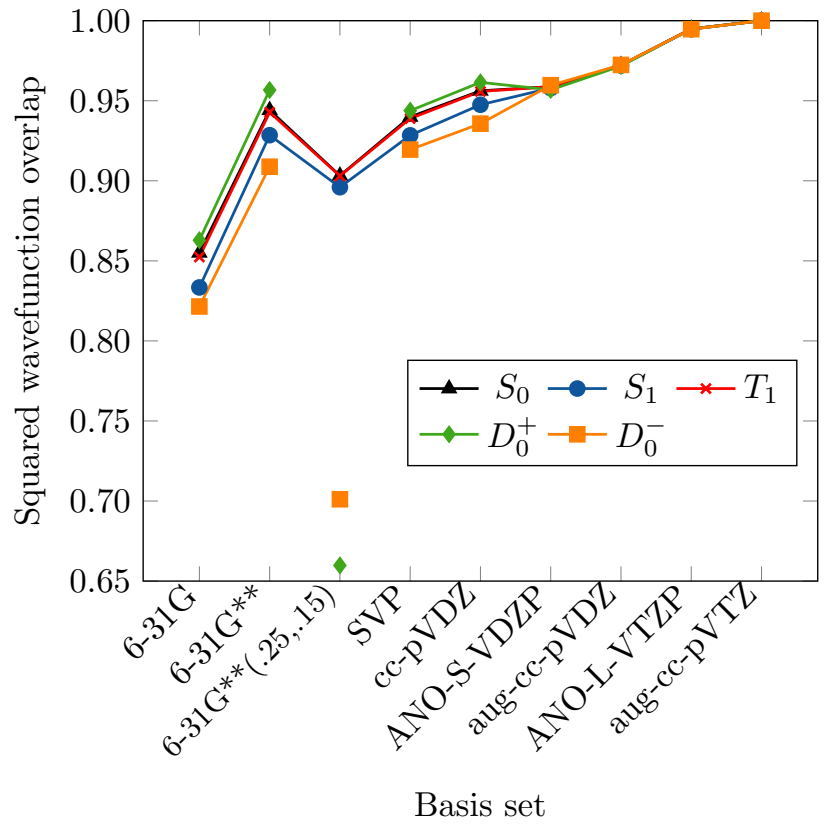

Figure 2: Squared overlap values of the CASSCF wavefunctions of the neutral states $S_{0}, S_{1}$, and $T_{1}$, as well as the ground states of the cation $D_{0}^{+}$and anion $D_{0}^{-}$of tetracene computed for various basis sets against the $\mathrm{CASSCF}$ / aug-cc-pVTZ reference.

of the cation $\left(D_{0}^{+}\right)$is described best while the agreement for the $\left(D_{0}^{-}\right)$state is worst. Among the neutral states, the $S_{1}$ description is worse compared to the $S_{0}$ and $T_{1}$. Out of the polarized double- $\zeta$ basis sets the worst agreement is found for the $6-31 \mathrm{G}^{* *}(0.25,0.15)$ basis set. The $S_{0}, S_{1}$, and $T_{1}$ are clustered around 0.90 whereas the $D_{0}^{+}$and $D_{0}^{-}$states are significantly lower at 0.66 and 0.70 , respectively. Figure 2 illustrates the advantage of the ANO-S-VDZP basis set from a different point of view: It does not only provide correct energies, but also the wavefunctions are described well providing a description that is consistent between all types of states and almost as good as the significantly larger aug-cc-pVDZ basis set.

Further insight into the properties of the wavefunctions is obtained by computing the RMS size of the electron density. The overall size $\left(\sigma_{\rho}\right)$ and its $z$-component are plotted in Figure 3 (a) and (b). Figure 3 (a) confirms the expected trends: The wavefunction of the anion $\left(D_{0}^{-}\right)$is most diffuse, with an RMS size of $3.562 \AA$ at the aug-cc-pVTZ level,

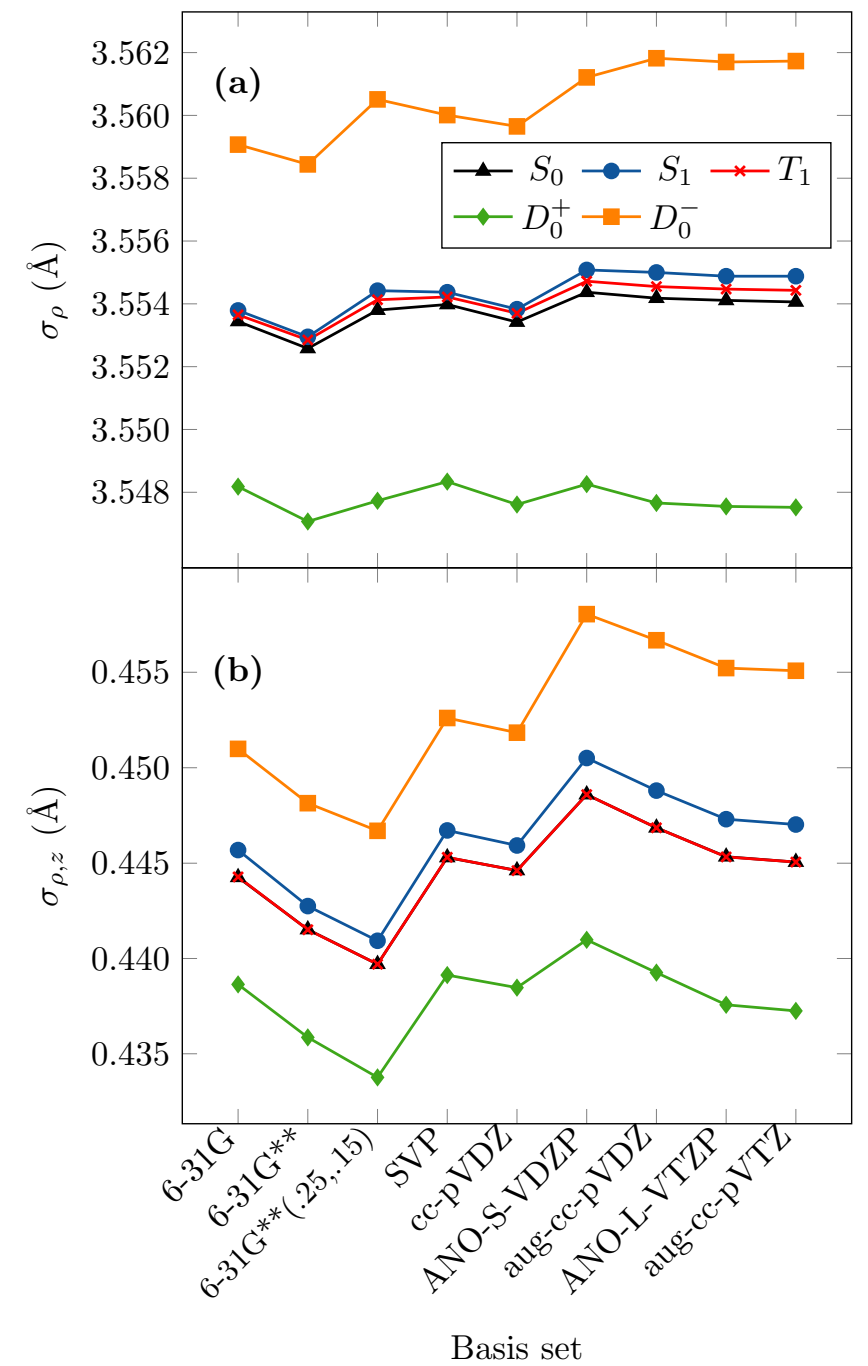

Figure 3: Spatial extent of different neutral and charged electronic states of tetracene computed at the CASSCF level for various basis sets: (a) overall value, (b) z-component. 
while the neutral states show intermediate values $(\sim 3.554 \AA)$ and the cation is most compact $(3.548 \AA)$. The effect of the basis set in Figure 3 (a) is rather small amounting to less than one tenth of a percent relative deviation for the overall RMS size. This insensitivity follows from the fact that the relevant differences disappear in two different averaging procedures: (i) averaging over all the electrons, and (ii) summing over the $x, y$, and $z$ coordinates. Whereas point (i) will be addressed below using the attachment/detachment density analysis, we address point (ii) here by analyzing only one Cartesian component of $\sigma_{\rho}$. The $x$ and $y$-components of $\sigma_{\rho}$, which extend along the molecular plane (cf. Figure 1), are to a large part trivially determined by the atomic positions whereas the $z$-component $\left(\sigma_{\rho, z}\right)$ directly measures how far the electron density extends into space away from the molecular plane. Therefore, $\sigma_{\rho, z}$ is chosen for further analysis, and it is plotted for the different states and basis sets in Figure 3 (b). In this case a somewhat different picture as opposed to the previous case is obtained. The $6-31 \mathrm{G}^{* *}(0.25,0.15)$ results are generally the lowest while the ANOS-VDZP results are the highest. This finding illustrates again that basis sets of the same form, i.e. polarized double- $\zeta$, can produce quite different wavefunctions and densities. Interestingly, the $6-31 \mathrm{G}^{* *}(0.25,0.15)$ basis set, which possesses specifically added diffuse basis functions, yields the most compact densities while ANO-S-VDZP, a basis set without explicit diffuse basis functions, yields the most diffuse densities. These findings show that it is not easy to predict the performance of a basis set based on qualitative considerations alone and highlight the importance of the interplay between the different basis functions. Another interesting observation is that the $S_{0}$ and $T_{1}$ states possess almost equivalent $\sigma_{\rho, z}$ values for all basis sets considered while the $S_{1}$ state is always notably more diffuse. This increase in diffuseness is in agreement with enhanced basis set effects for the $S_{1}$ state as observed in Figure 1. Finally, it should be noted that the lines in Figure 3 (b) are almost parallel. This means that despite the notable variations between the different ba- sis sets, the general trends are consistent.

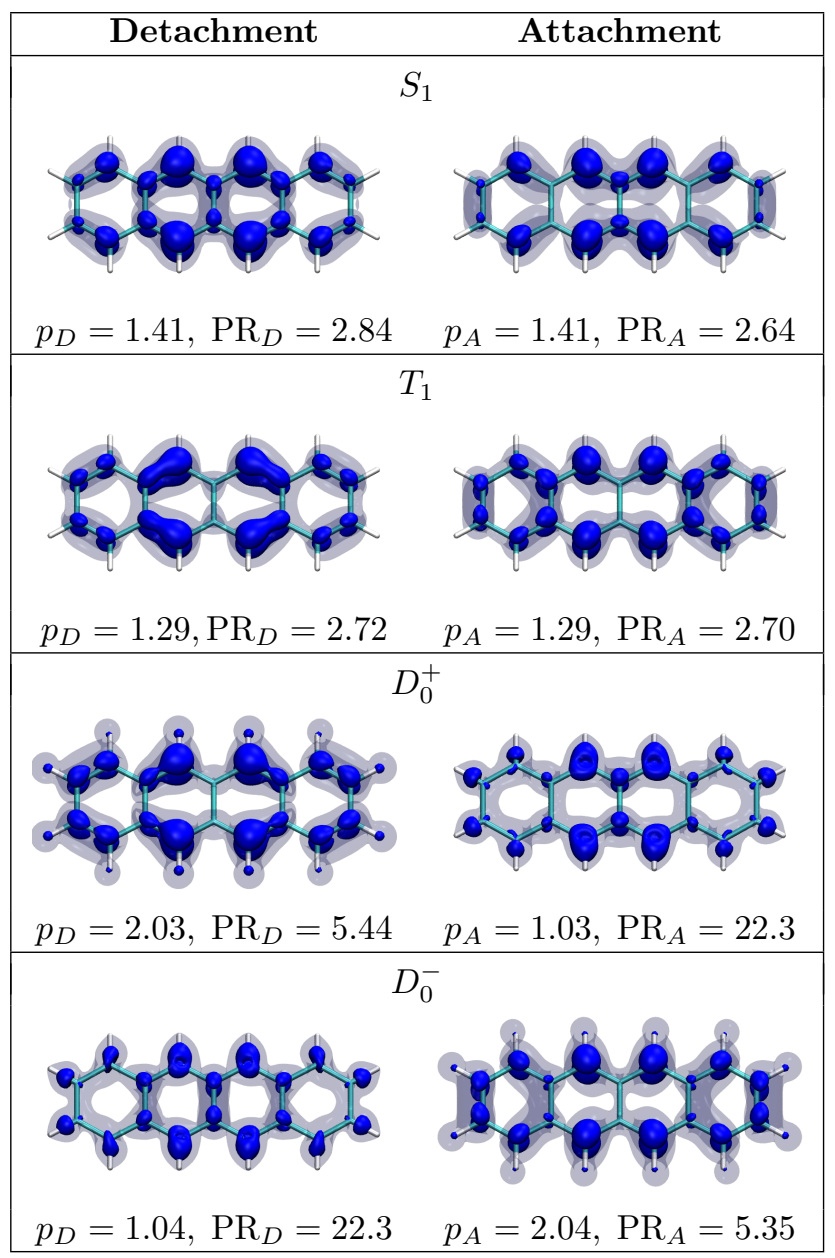

Figure 4: Attachment and detachment densities for different neutral and charged electronic states of tetracene computed at the CASSCF $(12,12) /$ aug-cc-pVTZ level of theory, isovalues: 0.004 (solid), 0.0013 (transparent).

A downside of the analysis of $\sigma_{\rho}$ is that it is computed as an average over all electrons including also the ones contained in core and $\sigma$ orbitals. Thus, from Figure 3 alone it is not possible to judge whether the discrepancy between the different basis sets derives from the orbitals active in the excitation and ionization processes or from inactive ones. To obtain a more detailed insight into the electrons active in the excitation and ionization processes, an attachment/detachment density analysis according to Eq. (5) is performed. Figure 4 shows the attachment and detachment densities of the $S_{1}, T_{1}, D_{0}^{+}$and $D_{0}^{-}$states computed with respect to the $S_{0}$ state. A decomposition of these 
densities into NDOs is given in the supporting Figures S1-S4. In a one-electron picture the detachment densities of the $S_{1}, T_{1}$ and $D_{0}^{+}$ states would all be the same, corresponding to the HOMO, while the one for the $D_{0}^{-}$state should vanish. Similarly, the attachment densities of the $S_{1}, T_{1}$ and $D_{0}^{-}$states should correspond to the LUMO while the one for the $D_{0}^{+}$ state should vanish. This is clearly not the case as all the attachment and detachment densities possess distinct features.

First, it is interesting to observe the promotion numbers (Eqs (10) and (11)), which count the total number of electrons rearranged. These amount to 1.41 and 1.29 in case of the singlet and triplet neutral excitations. In the case of the cationic state $D_{0}^{+}$the number of detached electrons $\left(p_{D}\right)$ amounts to 2.03 meaning that aside from the electron actually taken away, another 1.03 electrons are rearranged in the process. A very similar picture is found for the $D_{0}^{-}$ state. The important conclusion is that none of these processes should be understood as a simple one-electron process but that also the rearrangement of secondary orbitals plays a significant role. To count the number of the orbitals involved, we use the $\mathrm{PR}_{D}$ and $\mathrm{PR}_{A}$ measures defined in Eq. (12). In the case of the singlet and triplet states, these values are consistently around 2.7. This can be interpreted in the sense that in all cases there is one dominant NDO, i.e. the HOMO or LUMO, in addition to some secondary contributions from other $\pi$-orbitals (cf. Figures S1 and S2).

In the case of the $D_{0}^{+}$and $D_{0}^{-}$states the $\mathrm{PR}_{D}$ and $\mathrm{PR}_{A}$ values are significantly enhanced. This derives from the fact that also a large number of $\sigma$ orbitals are involved as can be seen in Figure 4 and more clearly in Figures S3 and S4, which show all the involved orbitals. The underlying process corresponds to a rearrangement of the electron density along the $\mathrm{CH}$ bonds, i.e. in the case of the cation the electrons are pulled inwards while they are pushed outwards for the anion.

From a technical perspective it is worth noting that the rearrangement effects are particularly apparent because the orbitals for each state are optimized individually. In the case of a common set of orbitals, created for example by Hartree-Fock or state-averaged CASSCF, such relaxation effects would have to be included in the form of orbital excitations. This requires sufficiently flexible wavefunction models and failing to do so can have detrimental effects. For example, it was found in the case of an iridium complex that state-averaged CASSCF systematically underestimated orbital relaxation accompanying metal-to-ligand CT transitions whereas the single-reference algebraic diagrammatic construction $\mathrm{ADC}(2)$ strongly overestimated it. ${ }^{24}$

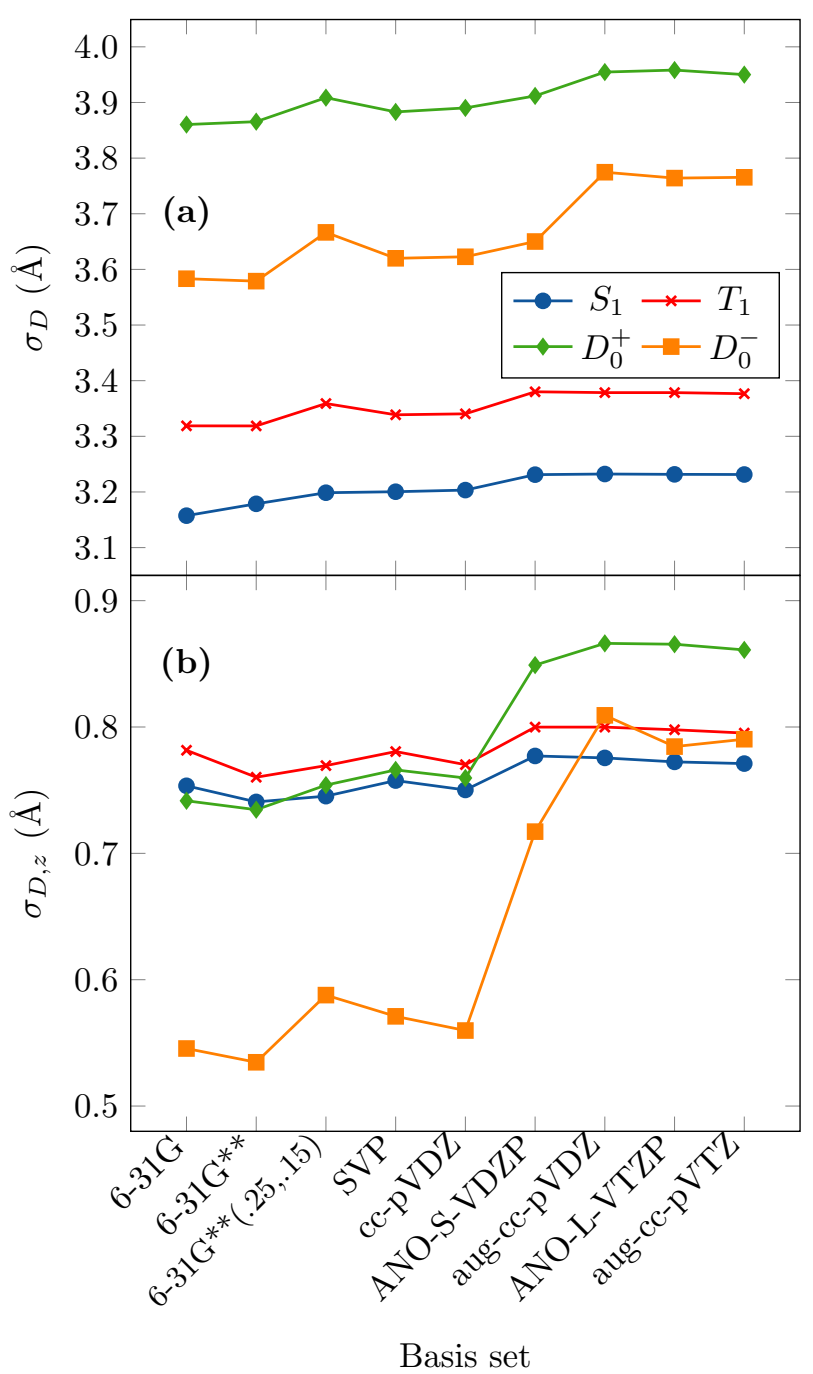

Figure 5: Spatial extent of the detachment densities of different neutral and charged electronic states of tetracene computed at the CASSCF level for various basis sets: (a) overall value, (b) z-component.

The variation of the promotion number $p_{A}$ 
with respect to the basis set is plotted in Figure S6 showing that this value stays fairly constant only increasing slightly with the more flexible basis sets. By contrast, the RMS sizes show some more notable alterations. This analysis in the case of the detachment density is presented in Figure 5. Considering, first, the spherically summed quantity $\sigma_{D}$, shown in Figure 5 (a), the general order $S_{1}<T_{1}<D_{0}^{-}<D_{0}^{+}$is observed for all basis sets. The values are fairly constant across the different basis sets and only in the case of the $D_{0}^{-}$state there is a spike for 6 $31 \mathrm{G}^{* *}(0.25,0.15)$ and a jump occurring between the ANO-S-VDZP and aug-cc-pVDZ basis sets. It should be noted, however, that the detachment density of the $D_{0}^{-}$state results entirely from orbital relaxation effects and is composed of a large number of individual NDO contributions [cf. Figure S4 (a)] and can thus not be easily interpreted.

A different vantage point is provided by viewing only the z-component [Figure 5 (b)], i.e. only the component of the density that extends away from the molecular plane. In this case, the jump in the value of the $D_{0}^{-}$state is more pronounced, going from $0.56 \AA$ (cc-pVDZ) through $0.72 \AA$ (ANO-S-VDZP) to about $0.80 \AA$ for the larger basis sets. A similar jump, occurring between the cc-pVDZ and ANO-S-VDZP basis sets is also observed for the $D_{0}^{+}$state. This jump indicates a qualitative change in the behavior: For the more compact basis sets, the $D_{0}^{-}$state is clearly separated from the rest, while in the case of the more diffuse basis sets it approaches the neutral states and the $D_{0}^{+}$state is separated. Interestingly, the ANO-S-VDZP basis can account (at least in part) for the increase in diffuseness observed for the larger basis sets.

The same analysis is presented for the attachment densities in Figure 6. The trends are very similar to those of Figure 5: in the case of the total RMS size $\sigma_{A}$ only slight increases with increasing basis set size are found and again a spike for $6-31 \mathrm{G}^{* *}(0.25,0.15)$ is seen. Stronger effects are again found for the $z$-component. Again, these values are significantly enlarged for the $D_{0}^{+}$and $D_{0}^{-}$states. An interpretation of these results is not straightforward owing to the

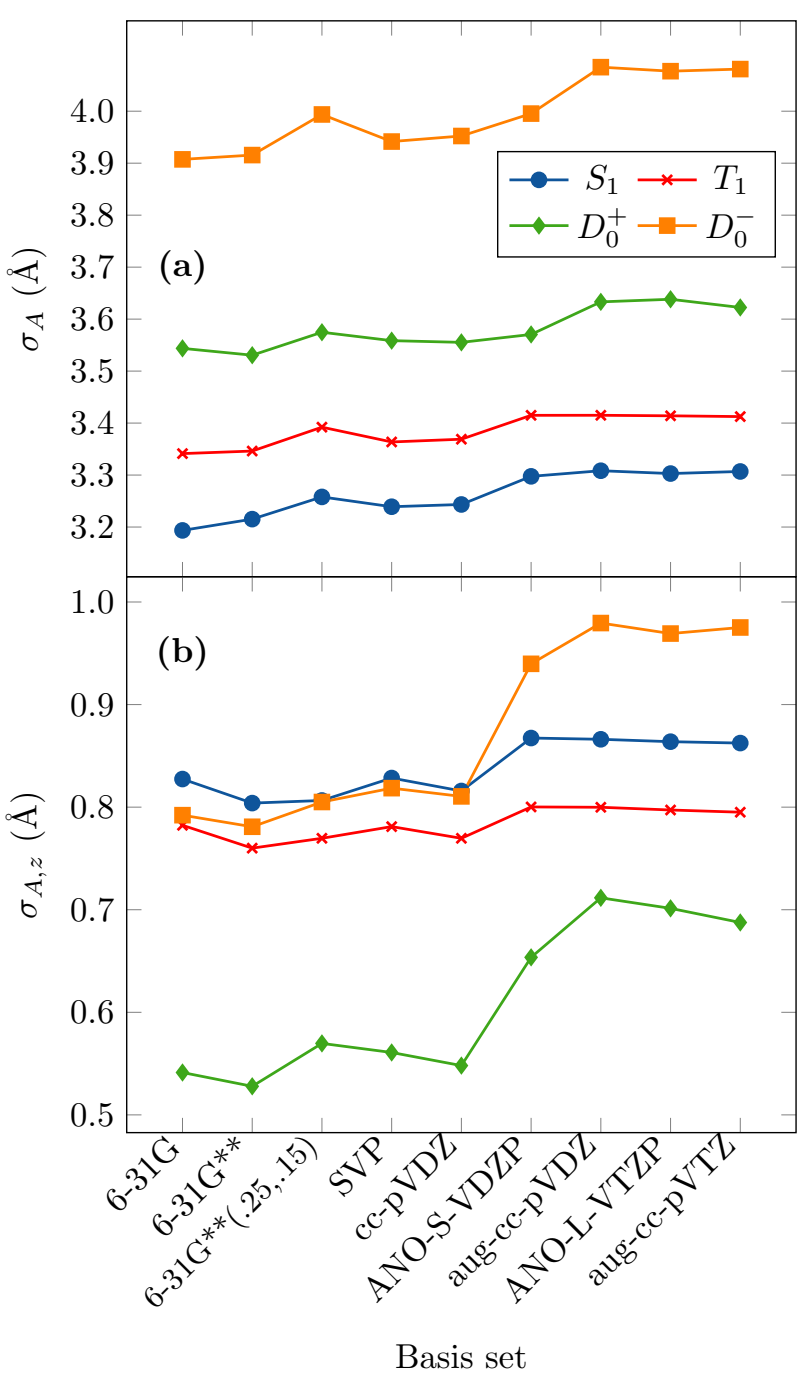

Figure 6: Spatial extent of the attachment densities of different neutral and charged electronic states of tetracene computed at the CASSCF level for various basis sets: (a) overall value, (b) z-component. 
fact that large numbers of orbitals are involved in the relevant detachment and attachment processes. However, an important general conclusion can be drawn: Ionization and electron attachment processes are more complex than one might presume at first sight, as they cannot be simply described by the removal/addition of one single electron from/to one orbital. A large number of orbitals are rearranged in the process and the precise description of this rearrangement requires large and flexible basis sets.

As a final comment it is fair to point out that not all properties of the wavefunctions are as sensitive to the basis set as the ones discussed above. For example, the effective number of unpaired electrons ${ }^{66}$ characterizing the natural orbital eigenvalue spectrum, which was used extensively in Refs 40,54,97 to characterize polyradical formation in polyacenes and related systems, is almost independent of the basis set for all five states considered here (Figure S7).

\section{Discussion}

The present study illustrates the challenges in describing different states of polyacenes. Not only dynamic correlation effects are important but also basis-set effects play a crucial role strongly affecting the different states. These challenges will certainly resurface in computations of dimers or larger aggregates, only that a detailed method comparison, such as performed here, is not feasible for such cases. Therefore, the results obtained here serve as a starting point for studies of processes such as charge transport, excitation energy transfer or singlet fission in polyacenes. Singlet fission is a particularly challenging process due to the fact that not only energies of the local singlet and triplet states have to be described well but also of the CT states in order to investigate their role in a superexchange mechanism. ${ }^{31,62,98}$ In this context, comparison of different computational methods (Table 2) reveals that an uncorrelated treatment will overestimate the $S_{1}$ energy by more than $1 \mathrm{eV}$ thus wrongly predicting an exothermic singlet fission process in tetracene
$\left(E_{\text {stt }}<-0.60 \mathrm{eV}\right)$ while at the same time placing the CT states too high in energy by $1 \mathrm{eV}$ $\left(E_{\text {gap }}=6.90 \mathrm{eV}\right)$. But even if dynamic correlation is included properly, an unfortunate choice of the basis set can move the relevant energies by $0.5 \mathrm{eV}$. This study showed, furthermore, that the variations in energies are accompanied by non-trivial variations in the wavefunctions.

An important result of this study was the superior performance of the ANO-S-VDZP basis set over other polarized double- $\zeta$ basis sets. This superiority was not only present for the energies but also in the cases of wavefunction overlaps and various density-matrix-based descriptors including the diffuseness of the wavefunctions. For many of the properties considered, a similar performance was found between the ANO-S-VDZP and aug-cc-pVDZ basis sets. Taking a closer look at the exponents of the primitive basis functions in these basis sets, it is observed that these are indeed similar. The major difference between these basis sets is that diffuse basis functions are added explicitly, i.e. as a contracted basis function containing only one primitive exponent, in the case of aug-cc-pVDZ while they are included implicitly in the contraction scheme of the ANO-SVDZP basis set. The present study thus highlights the importance of diffuse basis functions (cf. Refs 78,99). A detailed consideration of energies, densities, and wavefunctions suggests that for many properties it suffices to include diffuse functions implicitly in the contraction scheme (assuming that a proper parameterization is chosen).

\section{Conclusions}

The lowest singlet and triplet excited states of tetracene, as well as the ground state of its cation and anion were investigated using highlevel multireference methods in connection with detailed wavefunction analysis protocols. Different basis sets were investigated within the CASPT2 method finding a clear superiority of the ANO-S-VDZP basis set over other polarized double- $\zeta$ basis sets in the description of the energies as well as the overall wavefunction 
overlaps. Using a newly implemented wavefunction analysis toolbox in the program system MolCAS, the attachment and detachment densities were analyzed in detail, demonstrating, again, the superior performance of the employed ANO-S-VDZP basis set.

In a broader sense, this work illustrates that detailed wavefunction analysis protocols can provide new insight into the merits and shortcomings of the employed computational methods by illuminating intricate details of the computed wavefunctions. In the future, it will be interesting to study the influence of other wavefunction parameters such as the active orbital space and the level of dynamic correlation using similar analysis methods, and to compare the results of different quantum chemical methods.

Acknowledgement We thank S. Vancoillie and I. Fdez. Galván for technical help with respect to the Molcas implementation. This paper is based on work supported by the VSC Research Center funded by the Austrian Federal Ministry of Science, Research, and Economy (bmwfw) and by the Austrian Science Fund (FWF) project I2883-N34. SAM gratefully acknowledges funding from the Heidelberg Graduate School for Mathematical and Computational Methods for the Sciences. The computational results presented have been achieved in part using the Vienna Scientific Cluster (VSC), projects 70719 and 70726 .

\section{Supporting Information Avail- able}

Molecular coordinates (Table S1), natural difference orbitals of the different states (Figures S1-S4), plots of the total energies (Figure S5), promotion numbers (Figure S6), and the number of unpaired electrons (Figure S7). The input and output files of the CASPT2 $(12,12)$ calculations in connection with the wavefunction analysis module are provided as a zip archive.

\section{References}

(1) Roca-Sanjuán, D.; Aquilante, F.; Lindh, R. Multiconfiguration secondorder perturbation theory approach to strong electron correlation in chemistry and photochemistry. WIREs: Comp. Mol. Sci. 2012, 2, 585-603.

(2) Szalay, P. G.; Muller, T.; Gidofalvi, G.; Lischka, H.; Shepard, R. Multiconfiguration Self Consistent Field and Multireference Configuration Interaction Methods and Applications. Chem. Rev. 2012, 112, 108.

(3) Das, A.; Müller, T.; Plasser, F.; Krisiloff, D. B.; Carter, E. A.; Lischka, H. Local Electron Correlation Treatment in Extended Multireference Calculations: Effect of Acceptor-Donor Substituents on the Biradical Character of the Polycyclic Aromatic Hydrocarbon Heptazethrene. J. Chem. Theory Comput. 2017, 13, 2612-2622.

(4) Lee, J.; Small, D. W.; Epifanovsky, E.; Head-Gordon, M. Coupled-Cluster Valence-Bond Singles and Doubles for Strongly Correlated Systems: BlockTensor Based Implementation and Application to Oligoacenes. J. Chem. Theory Comput. 2017, acs.jctc.6b01092.

(5) Freitag, L.; Knecht, S.; Angeli, C.; Reiher, M. Multireference Perturbation Theory with Cholesky Decomposition for the Density Matrix Renormalization Group. J. Chem. Theory Comput. 2017, 13, 451459.

(6) Sharma, S.; Holmes, A. A.; Jeanmairet, G.; Alavi, A.; Umrigar, C. J. Semistochastic Heat-Bath Configuration Interaction Method: Selected Configuration Interaction with Semistochastic Perturbation Theory. J. Chem. Theory Comput. 2017, 13, 1595-1604.

(7) Lipparini, F.; Kirsch, T.; Köhn, A.; Gauss, J. Internally Contracted Multireference Coupled Cluster Calculations with 
a Spin-Free Dirac-Coulomb Hamiltonian: Application to the Monoxides of Titanium, Zirconium, and Hafnium. J. Chem. Theory Comput. 2017, 13, 3171-3184.

(8) Andersson, K.; Malmqvist, P. A.; Roos, B. O.; Sadlej, A. J.; Wolinski, K. Second-order perturbation theory with a CASSCF reference function. J. Chem. Phys. 1990, 94, 5483-5488.

(9) Szalay, P. G.; Bartlett, R. J. Approximately extensive modifications of the multireference configuration interaction method: A theoretical and practical analysis. J. Chem. Phys. 1995, 103, 36003612 .

(10) Kepenekian, M.; Robert, V.; Guennic, B. L. What zeroth-order Hamiltonian for CASPT2 adiabatic energetics of Fe(II)N6 architectures? J. Chem. Phys. 2009, 131, 114702.

(11) Zobel, J. P.; Nogueira, J. J.; González, L. The IPEA dilemma in CASPT2. Chem. Sci. 2017, 8, 1482-1499.

(12) Stein, C. J.; Reiher, M. Automated Selection of Active Orbital Spaces. J. Chem. Theory Comput. 2016, 12, 1760-1771.

(13) Plasser, F.; Wormit, M.; Mewes, S. A.; Thomitzni, B.; Dreuw, A. LIBwFA: Wavefunction analysis tool library for quantum chemical applications; available from https://github.com/libwfa/libwfa.

(14) Plasser, F.; Lischka, H. Analysis of Excitonic and Charge Transfer Interactions from Quantum Chemical Calculations. J. Chem. Theory Comput. 2012, 8, 27772789 .

(15) Plasser, F.; Wormit, M.; Dreuw, A. New tools for the systematic analysis and visualization of electronic excitations. I. Formalism. J. Chem. Phys. 2014, 141, 024106.

(16) Plasser, F.; Bäppler, S. A.; Wormit, M.; Dreuw, A. New tools for the systematic analysis and visualization of electronic excitations. II. Formalism. J. Chem. Phys. 2014, 141, 024107.

(17) Bäppler, S. A.; Plasser, F.; Wormit, M.; Dreuw, A. Exciton analysis of many-body wave functions: Bridging the gap between the quasiparticle and molecular orbital pictures. Phys. Rev. A 2014, 90, 052521.

(18) Plasser, F.; Thomitzni, B.; Bäppler, S. A.; Wenzel, J.; Rehn, D. R.; Wormit, M.; Dreuw, A. Statistical analysis of electronic excitation processes: Spatial location, compactness, charge transfer, and electron-hole correlation. J. Comp. Chem. 2015, 36, 1609-1620.

(19) Plasser, F. Entanglement Entropy of Electronic Excitations. J. Chem. Phys. 2016, $144,194107$.

(20) Mewes, S. A.; Mewes, J.-M.; Plasser, F.; Dreuw, A. Excitons in poly(para phenylene vinylene): A quantum-chemical perspective based on high-level ab initio calculations. Phys. Chem. Chem. Phys. 2016, 18, 2548-2563.

(21) Mewes, S. A.; Plasser, F.; Dreuw, A. Universal Exciton Size in Organic Polymers is Determined by Non-Local Orbital Exchange in TDDFT. J. Phys. Chem. Lett. 2017, 8, 1205-1210.

(22) Bohnwagner, M. V.; Burghardt, I.; Dreuw, A. Regular and red-shifted fluorescence of the donor-acceptor compound 5-(1H-pyrrole-1-yl)thiophenecarbonitrile (TCN) is efficiently quenched by internal modes of thiophene. Phys. Chem. Chem. Phys. 2017, 19, 13951-13959.

(23) Kautny, P.; Glöcklhofer, F.; Kader, T.; Mewes, J.-M.; Stöger, B.; Fröhlich, J.; Lumpi, D.; Plasser, F. Charge-transfer states in triazole linked donorâ $\breve{A}$ Şacceptor materials: strong effects of chemical modification and solvation. Phys. Chem. Chem. Phys. 2017, 19, 18055-18067. 
(24) Plasser, F.; Dreuw, A. High-Level Ab Initio Computations of the Absorption Spectra of Organic Iridium Complexes. $J$. Phys. Chem. A 2015, 119, 1023-1026.

(25) Wenzel, J.; Dreuw, A. Physical Properties, Exciton Analysis, and Visualization of Core-Excited States: An Intermediate State Representation Approach. J. Chem. Theory Comput. 2016, 12, 1314-1330.

(26) de Wergifosse, M.; Elles, C. G.; Krylov, A. I. Two-photon absorption spectroscopy of stilbene and phenanthrene: Excited-state analysis and comparison with ethylene and toluene. $J$. Chem. Phys. 2017, 146, 174102.

(27) Anthony, J. E. The larger acenes: Versatile organic semiconductors. Angew. Chem., Int. Ed. 2008, 47, 452-483.

(28) Hepp, A.; Heil, H.; Weise, W.; Ahles, M.; Schmechel, R.; von Seggern, H. LightEmitting Field-Effect Transistor Based on a Tetracene Thin Film. Phys. Rev. Lett. 2003, 91, 157406.

(29) Chu, C. W.; Shao, Y.; Shrotriya, V.; Yang, Y. Efficient photovoltaic energy conversion in tetracene-C 60 based heterojunctions. Appl. Phys. Lett. 2005, 86, 1-3.

(30) Schon, J. H.; Kloc, C.; Bucher, E.; Batlogg, B. Efficient organic photovoltaic diodes based on doped pentacene. Nature 2000, 403, 408-410.

(31) Smith, M. B.; Michl, J. Singlet fission. Chem. Rev. 2010, 110, 6891-936.

(32) Chan, W.-L.; Ligges, M.; Zhu, X.-Y. The energy barrier in singlet fission can be overcome through coherent coupling and entropic gain. Nat. Chem. 2012, 4, 840845.

(33) Congreve, D. N.; Lee, J.; Thompson, N. J.; Hontz, E.; Yost, S. R.; Reusswig, P. D.; Bahlke, M. E.; Reineke, S.; Voorhis, T.;
Baldo, M. A. External Quantum Efficiency Above $100 \%$ in a Singlet-ExcitonFission Based Organic Photovoltaic Cell. Science 2013, 340, 334-337.

(34) Lim, S. H.; Bjorklund, T. G.; Spano, F. C.; Bardeen, C. J. Exciton Delocalization and Superradiance in Tetracene Thin Films and Nanoaggregates. Phys. Rev. Lett. 2004, 92, 107402-1.

(35) Schuster, R.; Knupfer, M.; Berger, H. Exciton Band Structure of Pentacene Molecular Solids: Breakdown of the Frenkel Exciton Model. Phys. Rev. Lett. 2007, 98, 98-101.

(36) Sueyoshi, T.; Fukagawa, H.; Ono, M.; Kera, S.; Ueno, N. Low-density band-gap states in pentacene thin films probed with ultrahigh-sensitivity ultraviolet photoelectron spectroscopy. Appl. Phys. Lett. 2009, 95, 3-6.

(37) Grimme, S.; Parac, M. Substantial errors from time-dependent density functional theory for the calculation of excited states of large pi systems. ChemPhysChem 2003, 4, 292-5.

(38) Hajgató, B.; Szieberth, D.; Geerlings, P.; De Proft, F.; Deleuze, M. S. A benchmark theoretical study of the electronic ground state and of the singlet-triplet split of benzene and linear acenes. J. Chem. Phys. 2009, 131, 224321.

(39) Richard, R. M.; Herbert, J. M. TimeDependent Density-Functional Description of the L-1(a) State in Polycyclic Aromatic Hydrocarbons: Charge-Transfer Character in Disguise? J. Chem. Theory Comput. 2011, 7, 1296-1306.

(40) Horn, S.; Plasser, F.; Müller, T.; Libisch, F.; Burgdörfer, J.; Lischka, H. A comparison of singlet and triplet states for one- and two-dimensional graphene nanoribbons using multireference theory. Theor. Chem. Acc. 2014, 133, 1511. 
(41) Knippenberg, S.; Starcke, J. H.; Wormit, M.; Dreuw, A. The low-lying excited states of neutral polyacenes and their radical cations: a quantum chemical study employing the algebraic diagrammatic construction scheme of second order. Chem. Phys. 2010, 108, 2801-2813.

(42) Bettinger, H. F.; Tonshoff, C.; Doerr, M.; Sanchez-Garcia, E. Electronically Excited States of Higher Acenes up to Nonacene: A Density Functional Theory/Multireference Configuration Interaction Study. J. Chem. Theory Comput. 2016, 12, 305-312.

(43) Yang, Y.; Davidson, E. R.; Yang, W. Nature of ground and electronic excited states of higher acenes. PNAS 2016, 113, E5098-E5107.

(44) Sharifzadeh, S.; Darancet, P.; Kronik, L.; Neaton, J. B. Low-energy charge-transfer excitons in organic solids from firstprinciples: The case of pentacene. J. Phys. Chem. Lett. 2013, 4, 2197-2201.

(45) Ma, H.; Troisi, A. Direct optical generation of long-range charge-transfer states in organic photovoltaics. Adv. Chem. Phys. 2014, 26, 6163-6167.

(46) Modelli, A.; Mussoni, L.; Fabbri, D.; Ciamician, C. G.; Uni, V.; Selmi, V.; Alberto, V. S.; Ra, V. Electron Affinities of Polycyclic Aromatic Hydrocarbons by Means of B3LYP $/ 6-31+\mathrm{G}^{*}$ Calculations. J. Phys. Chem. A 2006, 110, 6482-6486.

(47) Horn, S.; Lischka, H. A comparison of neutral and charged species of oneand two-dimensional models of graphene nanoribbons using multireference theory. J. Chem. Phys. 2015, 142.

(48) Cornil, J.; Calbert, J. P.; Brédas, J. L. Electronic structure of the pentacene single crystal: Relation to transport properties. J. Am. Chem. Soc. 2001, 123, 12501251.
(49) Kubas, A.; Hoffmann, F.; Heck, A.; Oberhofer, H.; Elstner, M.; Blumberger, J. Electronic couplings for molecular charge transfer: Benchmarking CDFT, FODFT, and FODFTB against high-level ab initio calculations. J. Chem. Phys. 2014, 140, 14342-14354.

(50) Kubas, A.; Gajdos, F.; Heck, A.; Oberhofer, H.; Elstner, M.; Blumberger, J. Electronic couplings for molecular charge transfer: benchmarking CDFT, FODFT and FODFTB against high-level ab initio calculations. II. Phys. Chem. Chem. Phys. 2015, 17, 14342-14354.

(51) Nan, G.; Yang, X.; Wang, L.; Shuai, Z.; Zhao, Y. Nuclear tunneling effects of charge transport in rubrene, tetracene, and pentacene. Phys. Rev. B 2009, 79, 115203.

(52) Bendikov, M.; Duong, H. M.; Starkey, K.; Houk, K. N.; Carter, E. A.; Wudl, F. Oligoacenes: Theoretical Prediction of Open-Shell Singlet Diradical Ground States. J. Am. Chem. Soc. 2004, 126, 7416-7417.

(53) Pelzer, K.; Greenman, L.; Gidofalvi, G.; Mazziotti, D. A. Strong correlation in acene sheets from the active-space variational two-electron reduced density matrix method: effects of symmetry and size. $J$. Phys. Chem. A 2011, 115, 5632-40.

(54) Plasser, F.; Pašalic, H.; Gerzabek, M. H.; Libisch, F.; Reiter, R.; Burgdörfer, J.; Müller, T.; Shepard, R.; Lischka, H. The multiradical character of one- and two-dimensional graphene nanoribbons. Angew. Chem., Int. Ed. 2013, 52, 25812584 .

(55) Stein, C. J.; Reiher, M. Measuring MultiConfigurational Character by Orbital Entanglement. Mol. Phys. 2017,

(56) Luzanov, A. V.; Plasser, F.; Das, A.; Lischka, H. Evaluation of the quasi correlated 
tight-binding (QCTB) model for describing polyradical character in polycyclic hydrocarbons. The Journal of Chemical Physics 2017, 146, 064106.

(57) Siebbeles, L. D. A. Organic solar cells: Two electrons from one photon. Nat. Chem. 2010, 2, 608-609.

(58) Zimmerman, P. M.; Zhang, Z. Y.; Musgrave, C. B. Singlet fission in pentacene through multi-exciton quantum states. Nature chemistry 2010, 2, 648-652.

(59) Zimmerman, P. M.; Bell, F.; Casanova, D.; Head-Gordon, M. Mechanism for singlet fission in pentacene and tetracene: From single exciton to two triplets. J. Am. Chem. Soc. 2011, 133, 19944-19952.

(60) Feng, X.; Kolomeisky, A. B.; Krylov, A. I. Dissecting the effect of morphology on the rates of singlet fission: Insights from theory. J. Phys. Chem. C 2014, 118, 1960819617.

(61) Berkelbach, T. C.; Hybertsen, M. S.; Reichman, D. R. Microscopic theory of singlet exciton fission. III. Crystalline pentacene. J. Chem. Phys. 2014, 141, 074705.

(62) Tamura, H.; Huix-Rotllant, M.; Burghardt, I.; Olivier, Y.; Beljonne, D. First-Principles Quantum Dynamics of Singlet Fission: Coherent versus Thermally Activated Mechanisms Governed by Molecular $\pi$ Stacking. Phys. Rev. Lett. 2015, 115, 1-5.

(63) Fujihashi, Y.; Ishizaki, A. Fluctuations in Electronic Energy Affecting Singlet Fission Dynamics and Mixing with ChargeTransfer State: Quantum Dynamics Study. J. Ph 2016, 7, 363-369.

(64) Prlj, A.; Sandoval-Salinas, M. E.; Casanova, D.; Jacquemin, D.; Corminboeuf, C. Low-Lying $\pi \pi$ ấLU States of Heteroaromatic Molecules: A Challenge for Excited State Methods. J. Chem. Theory Comput. 2016, 12, 2652-2660.
(65) Mewes, S. A.; Plasser, F.; Dreuw, A. Communication: Exciton analysis in timedependent density functional theory: How functionals shape excited-state characters. J. Chem. Phys. 2015, 143, 171101.

(66) Head-Gordon, M.; Grana, A. M.; Maurice, D.; White, C. A. Analysis of Electronic Transitions as the Difference of Electron Attachment and Detachment Densities. J. Chem. Phys. 1995, 99, 14261-14270.

(67) Kannar, D.; Szalay, P. G. Benchmarking Coupled Cluster Methods on Singlet Excited States of Nucleobases. J. Mol. Model. 2014, 20, 2503.

(68) Roos, B. O.; Taylor, P. R.; Siegbahn, P. E. M. A complete active space SCF method (CASSCF) using a density matrix formulated super-CI approach. Chem. Phys. 1980, 48, 157-173.

(69) Ghigo, G.; Roos, B. O.; Malmqvist, P.-A. A modified definition of the zeroth-order Hamiltonian in multiconfigurational perturbation theory (CASPT2). Chem. Phys. Lett. 2004, 396, 142-149.

(70) Aquilante, F.; Pedersen, T. B.; Lindh, R. Low-cost evaluation of the exchange Fock matrix from Cholesky and density fitting representations of the electron repulsion integrals. J. Chem. Phys. 2007, 126, 194106.

(71) Hehre, W. J.; Ditchfield, R.; Pople, J. A. Self-Consistent Molecular Orbital Methods. XII. Further Extensions of GaussianType Basis Sets for Use in Molecular Orbital Studies of Organic Molecules. J. Chem. Phys. 1972, 56, 2257-2261.

(72) Hariharan, P.; Pople, J. The influence of polarization functions on molecular orbital hydrogenation energies. Theoretica chimica acta 1973, 28, 213-222.

(73) Kroon-Batenburg, L.; van Duijneveldt, F. The use of a moment-optimized DZP basis set for describing the interaction in the 
water dimer. Journal of Molecular Structure: THEOCHEM 1985, 121, 185 - 199.

(74) Hobza, P.; Šponer, J. Structure, Energetics, and Dynamics of the Nucleic Acid Base Pairs: Nonempirical Ab Initio Calculations. Chem. Rev. 1999, 99, 3247-3276.

(75) Schafer, A.; Horn, H.; Ahlrichs, R. Fully Optimized Contracted GaussianBasis Sets for Atoms Li to Kr. J. Chem. Phys. 1992, 97, 2571-2577.

(76) Dunning, T. H., Jr. Gaussian basis sets for use in correlated molecular calculations. I. The atoms boron through neon and hydrogen. J. Chem. Phys. 1989, 90, 1007-1023.

(77) Pierloot, K.; Dumez, B.; Widmark, P.O.; Roos, B. O. Density matrix averaged atomic natural orbital (ANO) basis sets for correlated molecular wave functions. Theoretica chimica acta 1995, 90, 87-114.

(78) Kendall, R. A.; Dunning Jr., T. H.; Harrison, R. J. Electron affinities of the firstrow atoms revisited. Systematic basis sets and wave functions. J. Chem. Phys. 1992, 96,6796 .

(79) Widmark, P.-O.; Malmqvist, P.-A.; Roos, B. O. Density matrix averaged atomic natural orbital (ANO) basis sets for correlated molecular wave functions. Theoretica chimica acta 1990, 77 , 291-306.

(80) Aquilante, F.; et al., Molcas 8: New capabilities for multiconfigurational quantum chemical calculations across the periodic table. J. Comp. Chem. 2016, 37, 506-541.

(81) Malmqvist, P.-A. k.; Roos, B. O. The CASSCF state interaction method. Chem. Phys. Lett. 1989, 155, 189-194.

(82) Vancoillie, S. Molpy ; available from https://github.com/steabert/molpy.

(83) Plasser, F. THEODORE: a package for theoretical density, orbital relaxation, and exciton analysis; available from http://theodore-qc. sourceforge . net/.
(84) Kohout, M. DGRID , version 4.6; Radebeul, Germany, 2011.

(85) Humphrey, W.; Dalke, A.; Schulten, K. VMD: Visual molecular dynamics. Journal of Molecular Graphics 1996, 14, 33 38.

(86) Plasser, F.; González, L. Communication: Unambiguous comparison of manyelectron wavefunctions through their overlaps. J. Chem. Phys. 2016, 145, 021103.

(87) Plasser, F.; Ruckenbauer, M.; Mai, S.; Oppel, M.; Marquetand, P.; González, L. Efficient and Flexible Computation of Many-Electron Wavefunction Overlaps. $J$. Chem. Theory Comput. 2016, 12, 1207.

(88) Richter, M.; Marquetand, P.; GonzalezVazquez, J.; Sola, I.; Gonzalez, L. SHARC: ab Initio Molecular Dynamics with Surface Hopping in the Adiabatic Representation Including Arbitrary Couplings. J. Chem. Theory Comput. 2011, 7, 1253-1258.

(89) Mai, S.; Richter, M.; Ruckenbauer, M.; Plasser, F.; Oppel, M.; Marquetand, P.; González, L. SHARC: Surface Hopping Including Arbitrary Couplings - Program Package for Non-Adiabatic Dynamics; available from http: //sharc-md .org. 2014 .

(90) Mai, S.; Marquetand, P.; González, L. A general method to describe intersystem crossing dynamics in trajectory surface hopping. Int. J. Quantum Chem. 2015, 115, 1215-1231.

(91) Dreuw, A.; Weisman, J. L.; HeadGordon, M. Long-range charge-transfer excited states in time-dependent density functional theory require non-local exchange. J. Chem. Phys. 2003, 119, 29432946 .

(92) Biermann, D.; Schmidt, W. Diels-Alder Reactivity of Polycyclic Aromatic Hydrocarbons. 1. Acenes and Benzologs. J. Am. Chem. Soc. 1980, 102, 3163-3173. 
(93) Tomkiewicz, Y.; Groff, R. P.; Avakian, P. Spectroscopic Approach to Energetics of Exciton Fission and Fusion in Tetracene Crystals. J. Chem. Phys. 1971, 54, 4504.

(94) Schmidt, W. Photoelectron spectra of polynuclear aromatics. V. Correlations with ultraviolet absorption spectra in the catacondensed series. J. Chem. Phys. 1977, 66, 828.

(95) Crocker, L.; Wang, T.; Kebarle, P. Electron affinities of some polycyclic aromatic hydrocarbons, obtained from electrontransfer equilibria. J. Am. Chem. Soc. 1993, 115, 7818-7822.

(96) Subotnik, J. E. Communication: Configuration interaction singles has a large systematic bias against charge-transfer states. J. Chem. Phys. 2011, 135, 071104.

(97) Das, A.; Müller, T.; Plasser, F.; Lischka, H. Polyradical Character of Triangular Non-Kekulé Structures, Zethrenes, p-Quinodimethane-Linked Bisphenalenyl, and the Clar Goblet in Comparison: An Extended Multireference Study. J. Phys. Chem. A 2016, 120, 1625-1636.

(98) Petelenz, P.; Pac, B. Is dipole moment a valid descriptor of excited state's chargetransfer character? J. Am. Chem. Soc. 2013, 135, 17379-86.

(99) Papajak, E.; Zheng, J.; Xu, X.; Leverentz, H. R.; Truhlar, D. G. Perspectives on basis sets beautiful: Seasonal plantings of diffuse basis functions. J. Chem. Theory Comput. 2011, 7, 3027-3034. 
Graphical TOC Entry

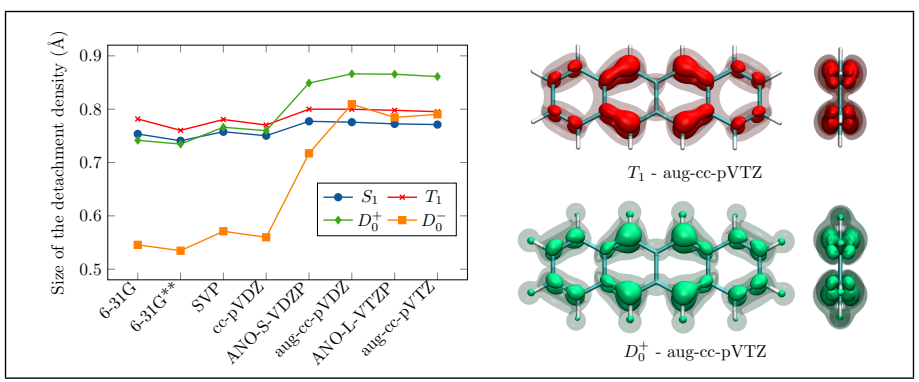

